\title{
Philosophiques
}

\section{Réponse aux commentaires de la « Disputatio »}

Race et culture en contextes

\section{Magali Bessone}

Volume 40, numéro 2, automne 2013

URI : https://id.erudit.org/iderudit/1023710ar

DOI : https://doi.org/10.7202/1023710ar

Aller au sommaire du numéro

Éditeur(s)

Société de philosophie du Québec

ISSN

0316-2923 (imprimé)

1492-1391 (numérique)

Découvrir la revue

Citer ce document

Bessone, M. (2013). Réponse aux commentaires de la " Disputatio " : race et culture en contextes. Philosophiques, 40(2), 487-496.

https://doi.org/10.7202/1023710ar d'utilisation que vous pouvez consulter en ligne.

https://apropos.erudit.org/fr/usagers/politique-dutilisation/ 


\title{
Réponse aux commentaires de la «Disputatio »' Race et culture en contextes
}

\author{
MAGALI BESSONE \\ UFR de philosophie, Université de Rennes 1 \\ magali.bessone-luquet@univ-rennesı.fr
}

\section{Le constructivisme est-il un anti-racisme?}

Capdevila et Jeffers rappellent la définition de la race que je donne dans une perspective constructiviste, en renvoyant, l'un au début, l'autre à la fin du chapitre 4, qui porte sur «la race comme construction sociale »; au début du chapitre, m'appuyant sur une définition proposée par Ron Mallon, je suggère de comprendre les races comme "des agrégats d'individus partageant des propriétés superficielles qui servent de critère pour une pratique sociale ou une ascription, ou [...] des groupes de personnes produits causalement ou institutionnellement par une telle pratique sociale» (87). En fin de chapitre, j'indique que le référent du concept de race dans les discours ordinaires est « un groupe fluctuant déterminé par des traits visibles fonctionnant comme des étiquettes faillibles associé à des traits déterminés sociologiquement et correspondant à une histoire continuée de domination et de conflits sociaux» (I I 4 ).

Capdevila estime que cette définition est trop lâche pour désigner de manière claire et distincte ce qu'on appelle ordinairement « race ». Pour préciser la définition, de sorte qu'elle corresponde mieux à nos usages ordinaires et qu'ainsi la dimension critique de la démarche constructiviste puisse effectivement s'appliquer, il affirme qu'il est indispensable de faire mention de la dimension biologique, au moins pour la désignation des "propriétés superficielles " ou des «traits visibles» partagés. Mais dans ce cas, déploret-il, le constructivisme ne peut éviter de se placer sous la dépendance du racisme, au point de devenir même, dans certains cas, un instrument commode du discours raciste - et ce d'autant plus que, par la référence à la biologie, c'est le non-biologique qui est visé, dans une tentative de naturalisation de toute différence.

Je souscris entièrement à l'opinion qui sous-tend la tension dénoncée par Capdevila, à savoir que la référence à la biologie pour définir les races (ou, d'ailleurs, pour affirmer leur inexistence) témoigne de la force d'une

1. Je voudrais remercier chaleureusement Nestor Capdevila, Luc Foisneau, Naïma Hamrouni et Chike Jeffers pour leur lecture attentive et leurs commentaires stimulants. Plusieurs de leurs questions se croisent, et j'ai choisi de procéder par regroupements thématiques, tout en privilégiant successivement dans la discussion le point d'entrée de l'un des commentateurs. 
idéologie positiviste qui croit voir dans la «science» l'outil privilégié, voire unique, capable de dire la "vérité " sur le sujet, et dont s'emparent ainsi racistes et anti-racistes, alors même que le concept de race n'a aucune existence biologique. Sur ce point, les analyses de François Jacob, notamment, énoncées dès $198 \mathrm{I}^{2}$, demeurent d'une extrême pertinence: ni la biologie en général ni la génétique en particulier n'ont quoi que ce soit à dire sur le concept de race, qui ne relève pas de leur domaine. Aussi dans une optique constructiviste, la mention biologique n'est-elle absolument pas requise, si ce n'est pour analyser comment les différences visibles ou propriétés superficielles en sont venues à être désignées comme tout particulièrement pertinentes pour classer des groupes d'êtres humains, parce qu'elles pouvaient apparaître comme validées par la science.

Or cette analyse fait d'ores et déjà partie de la déconstruction: elle est le premier pas pour saisir que la question "À quelle race appartient objectivement Susie Phipps? " n'a pas de sens si «objectif» signifie «biologique». On ne peut donc tenir rigueur au constructiviste de ne pouvoir y répondre —à moins d'accepter avec lui qu' " objectivement » signifie "dans le contexte socio-politique dans lequel Phipps se trouve", au sens que je définis p. IO2: " une fois que les conventions sont établies, que les pratiques sont routinisées, il y a des faits qui existent indépendamment des croyances individuelles ". Dans ce cas, la réponse du constructiviste est très claire: la race de Susie Phipps est celle qui lui est attribuée socialement et qui détermine un certain nombre de pratiques intersubjectives et institutionnelles. De plus, le constructivisme que je soutiens, soit un constructionnisme interactif inspiré de Ian Hacking, permet de rendre compte de la difficulté apparente du passing. Si l'on accepte de prolonger les analyses de Hacking par une définition du genre racial empruntée à Ronald Sundstrom (p. I05), il est aisé de comprendre que la race pour Phipps est moins réelle que pour d'autres individus de la même société: manque dans son cas l'un des trois éléments requis pour la production causale de son genre racial - en l'occurrence, la force "latérale ", correspondant aux normes attachées à la désignation et aux mises en pratique du genre par tous les membres de sa société. Le constructiviste peut donc répondre à la question et montrer pourquoi cette question semble complexe, sans mobiliser de référence à la biologie pour définir la race.

Demeure cependant, poursuit Capdevila, la possibilité d'un racisme constructiviste: le constructivisme n'est pas anti-raciste de manière inhérente ou constitutive de sa démarche. Cette affirmation ne me pose pas de problème: le constructivisme en effet est une méthode, et comme toute méthode elle peut être mise au service de valeurs et d'objectifs différents,

2. François Jacob, «Biologie et racisme», in «La science face au racisme», Le genre bumain, $\mathrm{n}^{\circ} \mathrm{I}, \mathrm{I} 98 \mathrm{I}$; voir aussi très récemment pour un renouvellement de la question, dénonçant l'illusion de l'apport de la génétique, Rasmus Grønfeldt Winther et Jonathan Michael Kaplan, "Ontologies and Politics of Biogenomic 'Race" ", Theoria, I36, sept. 2013. 
voire opposés (quelle qu'ait été l'histoire de ses usages jusqu'à présent). Toutefois, si cette méthode doit servir l'objectif de la construction de races telles qu'elles puissent être mobilisées dans des arguments racistes, et si le recours à la biologie n'est pas disponible, c'est un autre type de déterminant, la culture, qui sera utilisé —une culture essentialisée et naturalisée, racialisée. Capdevila l'admet, et c'est pourquoi, souligne-t-il, déplacer la race du côté de la culture, en atténuant la distinction de statut entre le discours racialiste et le discours culturaliste, ne résout, ni même n'euphémise le racisme. Capdevila situe le problème beaucoup plus fondamentalement dans le «scandaleux pouvoir discriminant de l'humanisme ", qui a produit historiquement, comme il le rappelle, de nombreux exemples de "racismes sans race ", où l'humanité, la culture, ou d'autres notions encore, nation, identité, ethnie, ont joué le rôle de la race.

J'admets très volontiers (je le reconnais p. I6 par exemple) que les termes qui fonctionnent dans les discours ordinaires actuels comme substituts de celui de race, "groupe ethnique » ou "culture » en particulier, ne sont pas de doux euphémismes, mais qu'ils ont produit, et produisent toujours, des effets de discrimination. J'admets également que l'humanisme est bien l'une de ces valeurs dont la modernité libérale et républicaine (pas plus que Foisneau je ne me préoccuperai ici de distinguer entre ces "cousins germainsde la pensée politique moderne ») a prétendu avoir l'apanage afin de justifier des rapports de domination, voire d'élimination de ceux qui étaient exclus du groupe des humains. J'y reviendrai. Contrairement à Capdevila, néanmoins, il me semble extrêmement douteux de se demander avec Cornélius de Paw s' "il aurait mieux valu persister dans l'opinion que les Américains étaient des singes, que de les reconnaître pour des hommes, et de s'arroger le droit affreux de les assassiner au nom de Dieu ». Cela me semble absurde d'abord parce que, comme l'ont montré récemment les insultes racistes à l'encontre de la Garde des sceaux française, Christiane Taubira, la «relégation" en dehors de l'humanité de certains groupes fonctionne toujours très bien du point de vue du racisme ordinaire: que l'opinion ait été renversée ou non, l'insulte demeure. Ensuite, et surtout, parce que singes ou hommes, s'il s'agissait d'assassiner les Américains pour s'approprier les terres et les richesses qu'ils détenaient, y compris leur force de travail, n'importe quel «droit» et n'importe quel «nom» pouvait faire l'affaire: si le scandale moral réside uniquement dans le fait que c'est «au nom de Dieu » que les assassinats ont été commis, alors certes, les assassiner en tant que singes au nom de la supériorité de l'espèce humaine sur les autres espèces animales considérées comme de simples ressources (Genèse, 2, I 8-20) est rendu moralement acceptable. Mais est-ce que c'est bien la seule invocation illégitime du nom de Dieu qui rend "affreux » le droit d'assassiner les Américains? L'humanisme ne me semble qu'un des déguisements parmi d'autres du discours d'exclusion, exactement au même titre, mais pas plus fondamentalement, que le racialisme ou le culturalisme, dès lors qu'il s'agit de 
désigner délibérément des groupes différenciés par des critères qui revêtent l'apparence de l'évidence pour mettre en place une relation de domination. Ce sont spécifiquement les modalités et les raisons de l'efficacité du discours racialiste qui m'ont arrêtée dans ce livre et qui expliquent les éléments définitionnels que j'ai retenus pour la notion de race.

J'admets toutefois, ce qui rejoint les remarques de Jeffers et de Hamrouni, être passée trop vite sur les rapports complexes entre race et culture, entre groupes raciaux et groupes culturels, sans avoir pris le temps d'examiner les différentes modalités de leurs intersections.

\section{La portée du multiculturalisme: déconstruction ou reconnaissance?}

Hamrouni remarque que «le rejet de la reconnaissance au profit de la seule déconstruction est radical» dans mon analyse et me demande si j'irais jusqu'à soutenir qu'aucune injustice ne peut se décliner en termes de nonreconnaissance. Je veux ici d'abord préciser que mon analyse ne porte que sur les groupes raciaux ou racialisés, et non pas sur les groupes qui peuvent subir des injustices pour des motifs de genre, de sexualité, de handicap, etc. $\mathrm{Si}$, bien entendu, des processus de domination fondés sur la désignation de ces différences peuvent se surajouter aux injustices pour motifs raciaux (comme le montrent notamment les études en termes d'insersectionnalité), il importe pour l'analyse de distinguer les différents processus, précisément parce qu'ils reposent sur une construction différenciée des catégories de justification des inégalités. Mon analyse dans le livre, et ma réponse ici, se limitent aux phénomènes raciaux, et je soutiens que dans leur cas en effet les phénomènes de disparités de pouvoir sont prépondérants.

C'est aussi ce que me reproche Jeffers. Il suggère qu'il faut accorder, davantage que je ne l'ai fait, une valeur positive aux processus d'identification et de différenciation culturelles à l'œuvre dans la catégorisation raciale. Selon lui - et c'est aussi ce qu'il soutient dans un article, paru en avril 2013 dans Ethics, en proposant une réinterprétation du texte "The Conservation of Races» de W. E. B. Du Bois-, il est moralement inacceptable d'affirmer comme je le fais que dans un monde idéal il n'y aurait pas de place pour la race. Alors que, dans ma perspective, la transformation du concept de race est un moyen en vue d'une fin qui demeure l'élimination des races, Jeffers estime qu'il faut procéder à l'envers. En plaçant au cœur de l'acception constructiviste de la race la dimension culturelle et en éliminant la race comme division politique, il devient légitime selon lui de viser une transformation du concept, permettant in fine une conservation des races-commecultures dans une société égalitaire: l'appartenance à ces groupes d'identité culturelle y serait débarrassée des processus externes d'essentialisation et des contraintes internes de conformité à l'authenticité, tout en donnant aux non-Blancs la chance de pouvoir s'associer librement au nom de valeurs culturelles partagées. 
La discussion à laquelle me convie Jeffers est passionnante et je crains de ne pouvoir y faire justice ici. Je voudrais cependant souligner trois points : premièrement, il n'est aucunement dans mon intention de nier la signification ou la valeur de groupes culturels «nonblancs» (pour reprendre la catégorie de Jeffers), à partir du moment en effet où la «culture» embrassée n'est ni un simple produit pour Blancs (quoique, bien entendu, tant que les Blancs possèdent la majeure partie du capital il ne soit en aucune manière absurde ou blâmable de profiter de la valeur marchande qu'ils accordent à l'exotisme ${ }^{3}$ ), ni figée par des relations de pouvoir non éliminées. Sur ce point, le jazz est un bon exemple, comme le mentionne Capdevila. Je me contenterai de renvoyer aux belles analyses de Frantz Fanon à ce sujet dans Les damnés de la terre ${ }^{4}$ : les "colonisateurs", se faisant "les défenseurs du style indigène ", s'indignèrent, écrit-il, lorsque le bebop succéda au blues, au motif que «le jazz ne doit être que la nostalgie cassée et désespérée d'un vieux nègre pris entre cinq whiskies, sa propre malédiction et la haine raciste des blancs ". "Il n'est pas utopique de supposer, poursuit-il, que dans une cinquantaine d'années, la catégorie jazz, cri hoqueté d'un pauvre nègre maudit, sera défendue par les seuls Blancs, fidèles à l'image stoppée d'un type de rapports, d'une forme de la négritude.» Contrairement à ce que défend Hamrouni, j'estime cependant que le multiculturalisme, notamment dans sa version libérale ${ }^{5}$, est une théorie politique particulièrement sensible à ce risque de fixation: Will Kymlicka propose une définition du groupe culturel idéalement rapportée à un concept de «culture sociétale» dont le contenu est très fermement substantiel; en outre, la relation entre les cultures est d'abord appréhendée à partir de l'exigence de protection contre la disparition ou l'assimilation (voir p. 202 et sq.).

À ce titre, et c'est mon deuxième point, il me semble difficile de séparer entièrement les races-comme-cultures du type de relations à l'œuvre dans une société: les groupes culturels sont des groupes relationnels, ou "positionnels ", pour mobiliser un terme proposé par Iris Marion Young ${ }^{6}$. La

3. Voir par exemple l'ironie mordante de Jamaica Kincaid à propos du tourisme dans l'île caribbéenne d'Antigua dans A Small Place, New York, Farrar, Strauss and Giroux, I988.

4. Frantz Fanon, Les damnés de la terre, in Euvres, Paris, La Découverte, p. 6I 8.

5. En réalité, loin de soutenir, comme m'en accuse Hamrouni, que Kymlicka a "une conception totalement an-historique des groupes sociétaux ", j'estime que le problème porte davantage sur le hiatus entre la contingence, voire le caractère ad hoc du critère historique mobilisé dans sa typologie des groupes (peuple autochtone, groupe d'immigration récente, groupe d'immigration installé, etc.) et la signification normative qui est attribuée à ce critère puisqu'il est le seul pertinent pour déterminer le type de droits légitimement exigés (droits à l'auto-gouvernement pour les minorités nationales, droits polytethniques pour les minorités ethniques).

6. Iris Marion Young, «Structural Injustice and the Politics of Difference ", in Anthony Simon Laden et David Owen dir., Multiculturalism and Political Theory, Cambridge, Cambridge University Press, 2007, p. 60-89. Je remercie Sophie Guérard de Latour d'avoir attiré mon attention sur ce point,en particulier dans le texte de son intervention « Multiculturalism 
mise en place d'une "politique de différence culturelle » comme celle préconisée par Jeffers, laquelle a pour objet de promouvoir la différence culturelle et de reconnaître pour les individus la valeur positive des affinités culturelles librement choisies, ne peut être menée indépendamment de la mise en place d'une "politique de différence positionnelle ", qui passe par la dénonciation préalable de la construction historique des groupes raciaux comme groupes injustement positionnés de manière structurelle. Il me semble que, selon la distinction proposée par Young, la proposition positive de Jeffers met l'accent sur des questions de liberté individuelle, tandis que mon problème réside dans la mise au jour d'inégalités structurelles. "Une société qui vise à redresser [l'inégalité racialisée structurelle] doit remarquer [notice] les processus de différenciation raciale avant de les corriger ", écrit Young (p. 7I): c'est bien ce que je me suis proposé de faire dans ce livre.

En d'autres termes, il est possible que la différence entre mes préconisations normatives et celles de Jeffers ne soit pas aussi radicale que le suggère Jeffers. D'une part, on peut sans doute à la fois chercher à mettre l'accent sur la mise en visibilité d'inégalités structurelles et insister sur l'importance, pour les individus, de la liberté d'association et de la célébration d'expériences positives partagées. Ainsi, je concède tout à fait à Hamrouni que, du point de vue des pratiques, les mesures de reconnaissance et les mesures de déconstruction ne sont pas nécessairement en opposition, mais qu'elles peuvent être "poursuivies simultanément», et même se renforcer mutuellement. D'autre part, il est possible d'envisager ces processus sur une échelle de temporalité différenciée: je suggère de considérer le concept de race sous l'angle de la construction sociale pour mettre au jour l'inégalité structurelle des groupes raciaux aujourd'hui, dans l'espoir que cette transformation conduise demain à l'élimination des races "comme divisions politiques", comme Jeffers et moi le souhaitons tous les deux. C'est sur la portée et l'interprétation de cette conséquence que porte en réalité l'essentiel de notre divergence: dans la mesure où mon concept de race est fondamentalement politique, je soutiens qu'il ne restera alors plus rien de «la race», et Jeffers estime qu'il restera alors la race-comme-culture.

Dès lors, il faut éclaircir ce que nous entendons tous deux par «culture» ainsi que le type d'intersections que nous envisageons entre les concepts de culture et de race. Or je crois - et c'est mon troisième point - que nos usages respectifs sont culturellement contextualisés. J'ai trouvé particulièrement frappant que Jeffers use de l'exemple des Noirs pour mettre l'accent sur la positivité d'un désir d'identification partagée des membres d'un groupe racial. Ce faisant, il affirme à la fois que les liens culturels de ce groupe ont une valeur et qu'ils peuvent permettre un renversement du stigmate historiquement associé au groupe. Or Jeffers ne soutient pas explicitement que la

and Anti-Racism, Allies or Enemies? " à la California Roundtable on Philosophy and Race, Chicago, IO-I 2 octobre 2013. 
valeur de l'identification à une culture noire réside dans le fait que la culture revendiquée représente le moyen et/ou le résultat «d'une histoire admirable de résistance ", du renversement du stigmate. Ainsi demeure une ambiguité sur ce qui doit être considéré comme une valeur en soi: la résistance à l'oppression, célébrée via la reconnaissance de la culture noire, ou toute culture en général, comme ensemble de croyances, de valeurs, de pratiques et de modes d'identification à respecter ?

Dans le premier cas, l'analyse demeure "politique ", et si la culture noire doit être promue, c'est au nom du renversement de positions ou de relations politiques entre des groupes hiérarchisés socialement, au nom du fait que la culture ici équivaut à un ensemble de valeurs et de pratiques

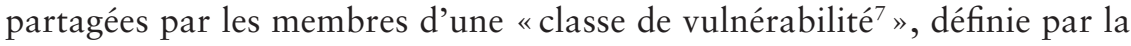
susceptibilité partagée à la domination - et à ce titre digne d'être soutenue. Si l'analyse peut alors s'étendre des Noirs aux non-Blancs dans nos sociétés (France, Canada, États-Unis) où les positions de domination sont occupées par les Blancs pour des raisons historiques continuées, si donc il est parfaitement légitime de l'appliquer aux musulmans, comme le fait Jeffers, ou aux Roms, comme le fait Foisneau, en tant que groupes racialisés, en revanche on peut alors difficilement l'étendre à toute race-commeculture, en particulier aux Blancs.

Dans le second cas, l'analyse est en effet "culturelle ", mais si c'est la culture en elle-même qui est bonne, indépendamment de l'histoire d'oppression qui a fourni, ascriptivement, les contours du groupe culturel, il devient difficile de discriminer entre les cultures à promouvoir et celles qui devraient susciter de la méfiance. Or je crois que c'est sur ce point que porte la différence de contexte entre l'acception de Jeffers et la mienne en ce qui concerne la culture. En effet, comme le souligne Jeffers, la littérature que je mobilise est anglo-saxonne, mais mon horizon de pensée, les problèmes que je cherche à résoudre et, en un mot, mes "ennemis ", ne sont pas les siens: de ce point de vue, je souscris à l'interprétation de Foisneau, qui perçoit bien que "[p]lus encore que le multiculturalisme dans ses variantes radicales et libérales (p.195-206), le républicanisme classique constitue en effet, dans le cadre de $[\mathrm{m}]$ a démonstration, une cible de choix».

\section{Républicanisme et culture républicaine}

Or, dans la perspective républicaine classique, les questions raciales ne sont pas traditionnellement abordées (lorsqu'elles le sont) sous les auspices de la race, ni même de la race-comme-culture, mais sous celles de la

7. Selon l'expression de Philip Pettit, I997; voir M. Bessone et S. Guérard de Latour, "Political, not Ethno-Cultural: a Normative Assessment of Roma Identity in Europe ", in G. Calder, M. Bessone et F. Zuolo, How Groups Matter?, Routledge, 20I4, p. I62-I8I. 
culture-comme-race ${ }^{8}$. En d'autres termes, c'est le concept de culture qui a servi en France d'outil privilégié pour construire les identités, et en particulier pour produire une conception de l'identité nationale comme groupe culturellement unifié. C'est pourquoi j'ai des réticences à considérer qu'il demeure au cœur du concept de race, une fois qu'on en a supprimé la dimension politique, un noyau «culturel» positif: d'une part (conceptuellement), en adoptant une approche politique du concept de culture, je suis convaincue qu'il n'existe pas de "culture noire " générique, mais des cultures spécifiques et différenciées, avec des langues, des religions, des coutumes et des visions du monde différentes, selon les contextes nationaux et les processus historiques de mise en relation (pacifique ou violente) des cultures ${ }^{9}$. D'autre part (politiquement), je crains que l'usage du concept de "culture» en France ne serve l'objectif politique inverse de celui que recherche Jeffers et qu'il ne fournisse aux conservatismes une justification supplémentaire du fondamentalisme culturel, qui demeure la forme dominante du racisme en France. Pour combattre cette forme spécifique, il me semble indispensable de rappeler qu'il y a "de la race", en dépit de son apparente absence, dans les usages français du concept de culture, et il me semble beaucoup moins adapté de soutenir qu'il y a «de la culture » dans les usages anglo-saxons du concept de race.

C'est ce contexte qui me conduit également au mouvement que Foisneau trouve discutable, et qui consiste à rejeter les «valeurs qui animent [le républicanisme] (universalisme et individualisme) » avec l'eau du bain du racisme, ou de "l'impuissance du républicanisme classique à venir à bout du racisme ». Là encore, la place me manque pour répondre à cette objection avec toute la précision qu'elle mérite, mais je tiens à faire deux (trop courtes) remarques. En premier lieu, l'universalisme et l'individualisme ne me semblent pas constituer des valeurs pour le républicanisme. Je les considère plutôt comme des instruments ou des principes théoriques, dont la "valeur» ne se mesure qu'à leur capacité de description et de prévision des comportements individuels et collectifs. Les valeurs explicitement revendiquées par le républicanisme classique, en revanche, sont l'égalité, la liberté et la fraternité.

En second lieu, l'universalisme et l'individualisme ne sont justement pas les meilleurs moyens de réaliser ces valeurs. En effet, ce sont des principes trop radicalement découplés des pratiques pour qu'ils aient la portée

8. David Beriss, "Culture-as-Race or Culture-as-Culture, Carribean Ethnicity and the Ambiguity of Cultural Identity in French society ", in Herrick Chapman et Laura L. Frader dir., Race in France, Interdisciplinary Perspectives on the Politics of Difference, New York, Berghahn Books, 2004, p. I II-I4O.

9. Sur ce point, les analyses menées par Fanon dans le chapitre 4, "Sur la culture nationale ", des Damnés de la terre (op. cit., p. 586-6I 2), me semblent extrêmement justes. 
transformatrice à laquelle ils prétendent ${ }^{10}$. En outre, sous la forme que ces principes ont revêtue dans l'histoire de la pensée républicaine française, ils ont fonctionné au cœur de discours de justification de stratégies de domination, servant à dissimuler que notre modernité politique repose sur la confusion entre la liberté comme propriété (entendue comme caractéristique d'une élite destinée à participer au contrat fondateur de la république) et la liberté égale pour tous comme droit fondamental de tout être humain ${ }^{11}$. L'individualisme, qui limite les effets de domination et de résistance à la somme des causes individuelles sans en dégager la dimension structurelle, ne permet pas de comprendre les luttes sociales - il est important de rappeler que le concept de domination n'a pas de sens lorsqu'il est rapporté à un individu, mais qu'il exige toujours la mise en place d'une logique globale, manifestée le cas échéant dans des relations intersubjectives ${ }^{12}$. L'universalisme, de son côté, a revêtu la forme d'une idéologie culturelle justifiant l'homogénéisation des valeurs et des modes de vie minoritaires. Cette idéologie est en France d'autant plus pernicieuse que si «la domination de certains groupes racialisés " n'est pas à strictement parler «imputable» à la "pensée des droits de l'homme » en général, le recours à la fiction abstraite de "l'homme » des droits de l'homme s'accommode très bien d'une substantialisation par un modèle empirique et historique particulier: le citoyen français. Ainsi, il est vrai, pour répondre à l'inquiétude de Foisneau, que je ne tiens pas particulièrement à sauver la culture républicaine, mais je ne tiens pas non plus à l' «accommoder à la mode américaine » — ce qui serait singulièrement incohérent avec l'épistémologie contextualiste qui est la mienne.

Dès lors, et je conclurai sur ce point, les interrogations de Hamrouni et de Foisneau prennent toute leur pertinence: "Quelles sont les implications politiques de l'approche théorique de la déconstruction?» demande Hamrouni. Comment, selon la formule puissante de Foisneau, "parvenir à reconstruire des normes communes sur les ruines laissées par la théorie critique de la race» ? Je ne peux ici que confirmer leur intuition: le travail reste à faire. Foisneau estime que si je tire toutes les conséquences de mon analyse, il ne restera plus de place pour le républicanisme; Hamrouni semble

10. Il m'est impossible ici de reprendre les analyses conduites par Cécile Laborde dans Critical Republicanism. The Hijab Controversy and Political Philosophy, Oxford, Oxford University Press, 2008: voir notamment sa dénonciation symétrique du rapport entre faits et normes dans le républicanisme classique et ce qu'elle nomme le républicanisme tolérant (le multiculturalisme): manque au premier une théorie sociale qui permette de mettre à nu les insuffisances de la république réelle par rapport à la république idéale; manque au second au contraire un système de normes permettant de théoriser l'empirique de manière satisfaisante.

11. Je renvoie ici à Eleni Varikas, "L'institution embarrassante», Raisons politiques, 2003, "Le corps du libéralisme», vol. I, p. 8 I-96.

12. Outre les analyses désormais classiques de Pettit, voir par exemple Ian Shapiro, "On Non Domination ", University of Toronto Law Journal, 32, 20I 2, p. 293-336 et, pour le cas spécifique qui nous occupe, Matthew Desmond/ Mustapha Emirbayer, "What is Racial Domination? ", Du Bois Review, 6, 2, 2009, p. 335-355. 
d'accord avec lui puisque, selon elle, l'abolition de la domination raciale que je vise «implique une certaine forme de "révolution" ". Plus précisément, Foisneau met en doute la possibilité de refonder une "république du respect »; Hamrouni suggère une piste (et je l'en remercie) du côté de la «responsabilité relationnelle ", notion qu'elle emprunte à Iris Marion Young, et qui permettrait de dépasser la dichotomie institution/individu dans la dénonciation, mais aussi dans la mise en place de critères pour évaluer les instances de manque de respect racial, ainsi que le type de défaut normatif (mauvais, injuste, vicieux) qu'elles expriment. Si je ne sauve pas la république, je peux donc peut-être sauver le respect égal. J'espère, en approfondissant les relations entre le respect et les "bases sociales du respect ", d'un côté, et l'exigence d'égalité et de responsabilité relationnelles, d'un autre côté, pouvoir répondre un jour à leur interrogation. 